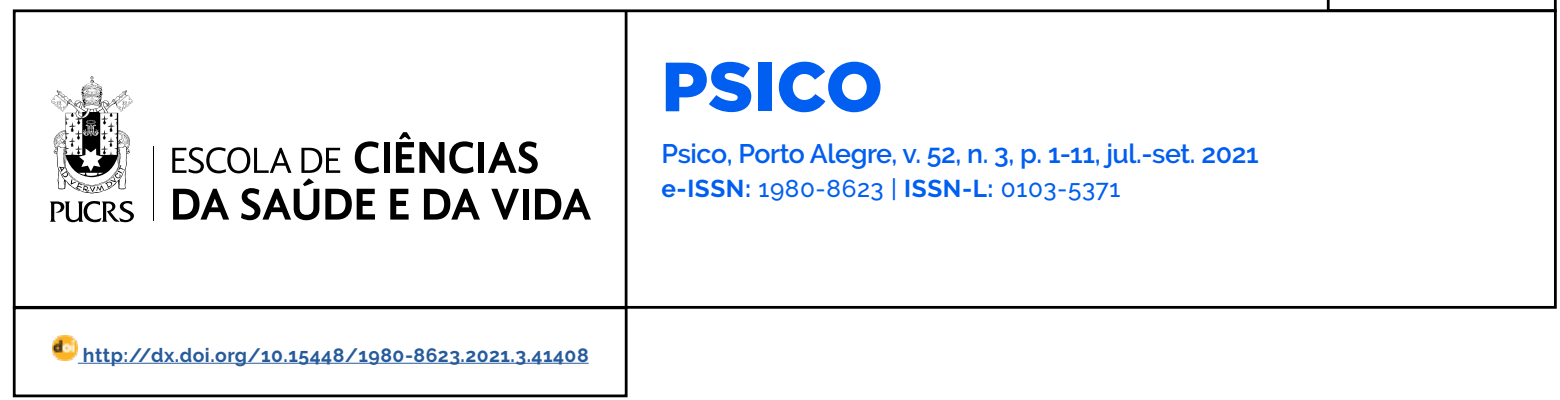

SEÇÃO: ARTIGO

\title{
Influência da percepção de risco sobre a COVID-19 no sofrimento psicológico dos profissionais de saúde
}

\author{
Influence of risk perception of COVID-19 on psychological distress of health professionals \\ Influencia de la percepción de riesgo del COVID-19 en el sufrimiento psicológico de los \\ profesionales de la salud
}

Priscila Goergen Brust-

Renck $^{1}$

orcid.org/0000-0001-9891-510X priscilabr@unisinos.br

\section{Jocieli Ferrari ${ }^{1}$ \\ orcid.org/0000-0001-5353-934X joci.ferrari@yahoo.com.br}

\section{Murilo Ricardo Zibetti ${ }^{1}$ \\ orcid.org/0000-0002-8934-5640 \\ murilozibetti@unisinos.br}

\section{Fernanda Barcellos}

\section{Serralta ${ }^{1}$}

orcid.org/0000-0003-4602-6495

fserralta@unisinos.br

Recebido em: 29 jul. 2021. Aprovado em: 5 ago. 2021. Publicado em: 27 out. 2021.

\section{(c) (1)}

Artigo está licenciado sob forma de uma licença Creative Commons Atribuição 4.0 Internacional.
Resumo: A saúde mental dos profissionais de saúde da linha de frente durante a pandemia da doença coronavírus 2019 (COVID-19) está relacionada a uma percepção qualitativa de risco sobre a doença, segundo a Teoria do Traço Difuso. Uma pesquisa de levantamento foi realizada com 134 profissionais de saúde da linha de frente em um municipio da região metropolitana do Rio Grande do Sul. Os participantes responderam a um questionário on-line sobre percepção de risco e a Clinical Outcome Routine Evaluation - Outcome Measure. Análises de regressão linear corroboraram a literatura, indicando que a percepção qualitativa do risco estava relacionada a menor sofrimento psicológico e uma avaliação quantitativa, a maior sofrimento. Os resultados demonstraram a importância da Teoria do Traço Difuso para compreensão dos riscos associados ao sofrimento mental (potencialmente clínico) de profissionais de saúde durante a pandemia. Palavras-chave: COVID-19, comportamentos de risco à saúde, avaliação de risco, promoção da saúde

Abstract: Mental health of frontline health professionals during the coronavirus disease 2019 (COVID-19) pandemic is related to a qualitative perception of risk about the disease, according to Fuzzy Trace Theory. A survey was carried out with 134 frontline health professionals in a city within the metropolitan area of Rio Grande do Sul. Participants answered an online questionnaire on risk perception and the Clinical Outcome Routine Evaluation - Outcome Measure. Linear regression analysis corroborated the literature, indicating that a qualitative perception of risk was related to less psychological distress and, a quantitative assessment, to greater suffering. Results showed the importance of Fuzzy-Trace Theory for understanding the risks associated with (potentially clinical) psychological distress of health professionals during the pandemic.

Keywords: COVID-19, health risk behaviors, risk assessment, health promotion

Resumen: La salud mental de los profesionales de la salud de primera línea, durante la pandemia de enfermedad por coronavirus 2019 (COVID-19), está relacionada con una percepción cualitativa de riesgo sobre la enfermedad, según la Teoría del Rasgo Difuso. Se realizó una encuesta a 134 profesionales de la salud de primera línea en un municipio de la región metropolitana de Río Grande del Sur. Los participantes respondieron un cuestionario en línea sobre percepción de riesgo y la Clinical Outcome Routine Evaluation - Outcome Measure. Los análisis de regresión lineal corroboraron la literatura, indicando que la percepción cualitativa del riesgo se relacionó con un menor sufrimiento psicológico y, una valoración cuantitativa, con un mayor sufrimiento. Los resultados demuestran la importancia de la Teoría del Rasgo Difuso para comprender los riesgos asociados con el sufrimiento mental (potencialmente clínico) de los profesionales de la salud durante la pandemia.

Palabras clave: COVID-19, conductas de riesgo para la salud, medición de riesgo, promoción de la salud 
Com o surgimento da doença coronavirus 2019 (COVID-19), no final de dezembro de 2019, os profissionais de saúde de linha de frente têm se exposto a muitos riscos de saúde. A sobrecarga que esses profissionais experimentaram no enfrentamento de uma doença desconhecida, com grande velocidade de propagação do vírus e alta taxa de contágio, em nivel mundial, levou ao aumento de problemas de saúde mental nesta classe laboral (Ruiz \& Gibson, 2020). Grande parte da dificuldade de enfrentamento da pandemia de COVID-19, no ano de 2020, se deve à fragilidade dos profissionais que apresentaram sintomas de sofrimento psicológico (Badahdah et al., 2020; Blake et al., 2021; Nacoti et al., 2020).

Simultaneamente ao aumento das informações sobre a doença, aumentaram também os casos no estado do Rio Grande do Sul, culminando no aumento das internações no final do ano de 2020 e no início de 2021. No entanto, a situação crônica de sofrimento mental dos profissionais de linha de frente levou ao aumento da discrepância na avaliação do risco da doença, que tem influência direta na adesão de medidas de promoção à saúde (Gold, 2020; Reyna, 2020b). Portanto, o presente estudo teve por objetivo avaliar a influência da percepção de risco do sofrimento psicológico dos profissionais de saúde da linha de frente, de um município da região metropolitana do estado do Rio Grande do Sul, no início do ano de 2021, quando o estado esteve em uma das situações mais críticas da pandemia (SES/RS, 2021).

\section{A pandemia e o profissional de saúde na linha de frente}

Os profissionais de saúde da linha de frente lidam diariamente com o risco de contaminarem-se com a COVID-19, ao tratar pacientes com suspeita ou confirmação de infecção pelo vírus. Eles também enfrentam a dificil tarefa de trabalhar muitas vezes sem insumos, com jornadas prolongadas, falta de equipamentos de proteção individual (EPIs) ou desconforto pela necessidade de seu uso, falta de vacinas, condições inadequadas de repouso e escassez de recursos humanos (Dong \& Bouey, 2020; Ornell et al., 2020). Por essa razão, esses profissionais podem sofrer maior vulnerabi- lidade aos impactos psicossociais da pandemia, sendo que o Brasil ocupa uma das primeiras posições no ranking do número de mortes (Conselho Federal de Enfermagem, 2021; Talevi et al., 2020).

Revisões sistemáticas e meta-análises sobre contextos de pandemias e epidemias, incluindo a COVID-19, demonstram o risco de aumento do sofrimento mental de profissionais de saúde, particularmente, aqueles que atuam diretamente no combate à doença (Serrano-Ripoll et al., 2020; Busch et al., 2021). Outros estudos referem que problemas mentais relacionados a distúrbios do sono, ansiedade, estresse pós-traumático, depressão e sindrome de burnout podem afetar especialmente os profissionais da linha de frente (Sani et al., 2020; Lai et al., 2020). Incertezas em relação ao futuro, longos períodos de isolamento, estigma social e insegurança no trabalho estão diretamente relacionados ao sofrimento psicológico, que tem intensificado sintomas emocionais prévios dos profissionais de saúde. Os desafios encontrados na prática cotidiana desses profissionais têm exacerbado questões emocionais já existentes antes da pandemia de COVID-19 e dificultado o enfrentamento da situação (Giorgi et al., 2020; Saidel et al., 2020). A vida dos profissionais da saúde pode ser afetada em todas as esferas, impactando o processo de recuperação daqueles que estão expostos a tantas adversidades. Muitos aspectos favorecem o desencadeamento de sintomas psicopatológicos, já que a vivência negativa é produzida por acontecimentos traumáticos, que são experimentados de forma particular (Lara et al. 2019). Dessa maneira, a promoção de sintomas nocivos pode ter consequências de importante gravidade, considerando o cenário de pandemia, nos serviços de saúde onde esses profissionais trabalham.

Problemas como cansaço físico e esgotamento mental, negligência institucional com relação às medidas de cuidado à saúde e pressão por atender as demandas impostas impactam diretamente no bem-estar psicológico dessa população, podendo influenciar na percepção de risco que os profissionais têm sobre a doença (Chung \& Yeung, 2020). Essa influência pode ser protetiva ou não, dependendo da maneira como os riscos são interpretados. Assim, a compreensão dos riscos 
a que os profissionais de saúde estão expostos colabora para que o comportamento adotado reduza os prejuizos da desproteção.

\section{Percepção de risco e sua influência na saúde mental dos profissionais de saúde}

O sofrimento apresentado pelos profissionais de saúde que vivenciam situações trágicas está relacionado, entre outras coisas, à percepção de risco referente a essas condições de saúde e doença. Conforme a visão da Teoria do Traço Difuso (Reyna \& Brainerd, 2011), a percepção de risco sobre a mudança deve ser entendida de forma subjetiva, orientada pela compreensão dos fatos, a partir de determinada realidade nos quais se encontram e na experiência prévia do observador. De acordo com essa teoria, as pessoas pensam no risco baseado em dois processos paralelos, a saber (1) fundamentado em uma representação qualitativa (que resume a essência do problema) e (2) baseado em uma representação quantitativa (que descreve de maneira literal a situação), independentemente do contexto. A preferência por um ou outro processo para interpretar o risco de contaminação pela doença (i.e., baseado na essência ou no literal) está diretamente relacionado ao comportamento de promoção da saúde ou busca ao risco (Brust-Renck et al., 2016; Reyna, 2012). Por um lado, as pessoas que analisam o risco qualitativamente (com base no entendimento de essência do problema) tendem a uma menor exposição a essas situações, pois julgam a prevenção de riscos como medida protetora. Por outro lado, aqueles que analisam quantitativamente o risco (com base na representação literal), ao contrabalançar riscos e beneficios, por meio da identificação que os beneficios de um evento isolado possam superar os riscos, estão mais propensos a comportamentos de exposição a situações que envolvem risco (Mills et al. 2008).

Com base nessa abordagem teórica, as mudanças e dificuldades vivenciadas pelo profissional de saúde em sua vida, a partir do cenário da pandemia, podem estar relacionadas a benefícios ou prejuizos na sua saúde. Ao perceber o risco de forma qualitativa, como alto e perigoso, bem como reconhecer o efeito protetor das recomendações de cuidados à saúde, o profissional de saúde tende a adotar medidas de proteção adequadas, como uso de EPIs, cuidados de higiene e segurança. Em contrapartida, uma percepção literal do risco de exposição à COVID-19 como provável nos próximos meses, independentemente do uso de medidas de proteção, reconhecendo que pode ser infectado em algum momento, contribui para menor adesão às medidas protetivas (Reyna, 2020a).

Como a desesperança está relacionada à representação mental literal de "vou me contaminar de qualquer forma nos próximos meses, não importa o que eu faça", o profissional passa a se sentir mais ansioso e inseguro frente a exposição ao risco de si e de sua familia. Já a percepção do risco baseada em um processamento de essência (qualitativo) está associada a uma noção de "basta apenas uma vez sair sem máscara para pegar o virus, melhor evitar todo risco da COVID-19, que poderia me deixar gravemente doente", frente a qual o profissional de saúde passa a se sentir menos ansioso e mais seguro. Segundo a Teoria do Traço Difuso, portanto, a forma como as pessoas percebem o risco de contaminação da COVID-19 parece definir a conduta de atenuação desse risco, reconhecendo a existência de medidas protetivas e a necessidade de adesão aos cuidados (Reyna, 2020b).

\section{Método}

No presente trabalho, foi realizado um estudo de levantamento para a avaliação da percepção de risco de contaminação da COVID-19 pelos profissionais da saúde da linha de frente, tanto em termos de representação de essência e literal. As diferentes formas de processamento da informação serão utilizadas para predizer o sofrimento psicológico. A hipótese é que o endosso dos comportamentos de essência leva a maior bem-estar psicológico desses profissionais, enquanto o dos comportamentos literais aponta para um possivel sofrimento emocional, conforme a Teoria do Traço Difuso.

\section{Participantes}

Participaram do estudo 134 trabalhadores vinculados, diretamente ou contratualizados, à 
Secretaria de Saúde de um municipio da região metropolitana do estado do Rio Grande do Sul, Brasil. Eram potenciais respondentes ao levantamento aproximadamente 2.000 trabalhadores. Dentre os que responderam, a maioria era do sexo feminino (83,6\%; n=112), com idades entre 23 e 64 anos (Média = 40,46; $D P=10,28$ ). A maior parte dos participantes encontrava-se casado ou com união estável (49,3\%; n=66), no momento da pesquisa, e 30\% estavam realizando acompanhamento psiquiátrico ou psicológico. Cerca de $37 \%$ dos participantes testaram positivo para COVID-19 até o momento da pesquisa. Todos os participantes responderam ao questionário na integra.

\section{Instrumentos}

O questionário foi constituido por três tipos de medidas, contendo dados sociodemográficos, a avaliação de sofrimento psicológico como desfecho clínico em saúde, e escalas de percepção de risco. A primeira parte do levantamento consistiu em uma ficha de dados sociodemográficos e de saúde, desenvolvido pelos pesquisadores exclusivamente para essa pesquisa, com a finalidade de coletar informações demográficas (idade, estado civil, gênero) e a respeito da saúde do paciente (histórico psiquiátrico e contaminação com a doença).

Um segundo instrumento respondido pelos participantes foi o Clinical Outcome Routine Evaluation - Outcome Measure (CORE-OM), desenvolvido por Evans et al. (2000, 2002) para avaliação de desfechos clinicos de saúde mental. Trata-se de um questionário de autorrelato, com 34 itens, que avalia a frequência com a qual o participante experimenta aspectos relacionados ao sofrimento psicológico em uma escala tipo Likert de 5 pontos, que varia de o (nunca) até 4 (sempre). Os itens podem ser agrupados em quatro dominios: bem-estar subjetivo (BES); problemas e sintomas (PS); funcionalidade diária (FD), e risco (R). Os itens dos domínios BES, PS, e FD, comumente são agrupados para avaliação do sofrimento mental em uma escala denominada Não Risco (com a soma de 28 itens). Os outros seis itens compõem a escala $\mathrm{R}$, avaliando pensamentos ou comportamentos de agressão para si e para os outros. Essa subescala é muito frequentemente tratada de forma categórica, ou seja, o endosso a qualquer item é considerado indicativo de presença de risco. Pontuações altas são indicadores de maior sofrimento mental dentro de cada domínio. A versão utilizada no presente estudo foi adaptada para o português brasileiro por Santana et al. (2015), conforme as diretrizes do CORE System Trust (www.coresystemtrust.org.uk/cst-translation-policy recuperado em 25 de julho de 2021). A consistência interna da versão original (Evans et al., 2002) é considerada adequada ( $\alpha$ de Cronbach $=0,94$ ), bem como na presente amostra ( $\alpha$ de Cronbach $=0,95$ ).

Por fim, foram realizadas medidas de percepção de risco, elaboradas especificamente para a presente pesquisa com base na Teoria do Traço Difuso (e.g., Mills et al., 2008) e que podem ser agrupadas em três subescalas: Riscos e Beneficios Globais, Principios de Essência e Risco (Literal) Especifico. Para uma avaliação global da doença, duas perguntas foram incluidas: a primeira sobre riscos ("De acordo com o que você pensa sobre seu comportamento frente a pandemia, qual das opções a seguir melhor representa o RISCO de contágio da COVID-19?") e a segunda sobre beneficios dos usos de EPIs contra COVID-19. Ambas foram escritas no mesmo formato para identificar compreensão global, utilizando uma escala Likert de 4 pontos, variando de o (nenhum) a 3 (alto). A escala de Principios de Essência foi utilizada para avaliar a representação qualitativa do risco individual frente à situação da COVID-19, por meio de seis perguntas como: "Melhor se proteger do que arriscar o contágio da COVID-19". As perguntas sobre Risco Especifico de se contaminar com a COVID-19 (e.g., "É provável que eu pegue a COVID-19 no próximo mês") avaliam a representação literal, por meio de quatro perguntas. Foi utilizada uma escala Likert de 5 pontos, variando de 1 (discordo totalmente) a 5 (concordo totalmente), para indicar aceitação do conteúdo das afirmativas. A consistência interna pode ser considerada aceitável para avaliar um construto teórico, em escalas não-padronizadas ( $\alpha$ de Cronbach $=0,56$ e 0,87, para Princípios de Essência e Risco Especifico, respectivamente). Pontuações mais altas refletiram maior percepção de risco de essência ou literal. 


\section{Procedimentos de coleta de dados}

A coleta foi realizada entre março e abril de 2021, por meio da aplicação de um questionário online, com base no Microsoft Forms. Primeiramente, foi enviado e-mail institucional para todos os servidores da saúde, com convite para participação na pesquisa e o link do formulário. O convite foi reforçado após o quinto dia do envio e, também, por vídeos divulgados em redes sociais. Ao acessar o formulário, os participantes visualizavam o Termo de Consentimento Livre e Esclarecido e, uma vez expressando concordância, passavam a responder ao questionário.

\section{Procedimentos de análise de dados}

Primeiramente, a frequência com a qual os participantes experienciam sofrimento mental, segundo a CORE-OM, foi avaliada por meio de uma análise de regressão linear múltipla, utilizando o escore total como variável dependente (com a pontuação mais alta indicando maior nivel de sofrimento) e os escores das escalas de avaliação de representação qualitativa (Principios de Essência) e quantitativa (Risco Especifico) do risco de contaminação por COVID-19, como variáveis preditoras. Acrescentou-se no modelo, para finalidade de controle, as variáveis: (1) diagnóstico positivo para COVID-19 (Não = 1; possivelmente, mas sem teste $=2$; e im, com teste $=3$ ) e (2) presença de sofrimento psicológico significativo (Não = 0; Sim = 1). A mesma análise foi realizada para cada um dos domínios BES, PS, FD, e R.

Em um segundo momento, foi avaliada a predição de variáveis demográficas e da percepção de risco para a classificação no grupo com maior sofrimento psicológico. Foi realizada uma análise de regressão logistica binária e hierárquica de dois blocos, utilizando o método Enter, para criar um modelo preditivo dos participantes com sofrimento psicológico significativo e endosso a risco. Essa regressão visava avaliar a apresentação, concomitantemente, de resposta de risco e indicadores de alto sofrimento mental. No primeiro passo da análise foram incluídos potenciais preditores demográficos e de saúde (gênero, idade, estado civil, e histórico de atendimento psiquiátrico ou psicológico). Já no segundo passo, foram inseridas as variáveis de avaliação geral e especifica de riscos de contágio e benefícios do uso de EPIs no contexto do trabalho durante a pandemia.

\section{Aspectos éticos}

O projeto foi submetido e aprovado pelo Comitê de Ética (CAAE\#31225520.0.0000.5344). Além disso, o projeto foi realizado com anuência e suporte do local em que a pesquisa foi realizada, por estar em acordo com as diretrizes das pesquisas com seres humanos, previstas na Resolução n ${ }^{\circ}$ 466/2012 e n 510/2016 do Conselho Nacional de Saúde $(2012,2016)$ e na Resolução n 010/12 do Conselho Federal de Psicologia (2012). Os participantes que concordaram em participar, assinaram o Termo de Consentimento Livre e Esclarecido. Todos os respondentes que permitiram contato após o questionário receberam retorno de seu padrão de respostas e puderam acessar cartilhas especificas sobre saúde mental na pandemia. Os participantes em classificados como em sofrimento receberam suporte e encaminhamento para a rede de saúde mental.

\section{Resultados}

Percepção de risco como preditor de sofrimento psicológico

A média e o desvio padrão das escalas de desfecho e preditoras estão apresentadas na Tabela 1. De forma geral, conforme interpretação dos valores, os participantes identificaram o Risco Global de contágio da COVID-19 com base no seu comportamento individual como "médio", e o Beneficio Global de usar EPIs como "alto". Além disso, parecem concordar majoritariamente com Princípios de Essência e discordar (de formal parcial) ou manter-se neutro frente à percepção de Risco Específico. Os resultados das análises de regressão múltipla (Tabela 2), para predizer o nivel de sofrimento psicológico, com base no escore total da escala CORE-OM, apresentou indices adequados de ajuste $[F(130,4)=33,53, p<0,001$; $\left.\mathrm{R}^{2}=0,50\right]$. As variáveis preditoras de maior sofrimento psicológico foram o diagnóstico positivo 
da COVID-19 e o Risco Especifico, sugerindo que a avaliação quantitativa do risco, bem como receber diagnóstico da COVID-19, foram responsáveis por problemas de saúde mental. Os resultados também indicaram que a representação qualitativa do risco foi preditora de menor sofrimento psicológico.

Tabela 1 - Médias e Desvios-Padrão para as Escalas do CORE-OM, Avaliação Global de Riscos e Benefícios, Principios de Essência, e Risco Específico

\begin{tabular}{lcc}
\hline & Média & $\begin{array}{c}\text { Desvio } \\
\text { Padrão }\end{array}$ \\
\hline CORE-OM Escore Total & 1,27 & 0,61 \\
Bem-Estar Subjetivo & 1,81 & 0,82 \\
Problemas e Sintomas & 1,69 & 0,80 \\
Funcionalidade Diária & 1,29 & 0,70 \\
Risco & 0,27 & 0,46 \\
Percepção de risco & & \\
Risco Global & 1,97 & 0,88 \\
Benefícios Global & 2,84 & 0,41 \\
Princípios de Essência & 4,79 & 0,35 \\
Risco Especifico & 2,64 & 0,94 \\
\hline
\end{tabular}

Os demais domínios do CORE-OM mantiveram um padrão semelhante de resultado, com diagnóstico positivo da COVID-19 sendo o principal preditor de sofrimento psicológico em todos os domínios, apresentando os maiores coeficientes de regressão padronizado. As escalas baseadas na Teoria do Traço Difuso foram preditoras de variância, mesmo quando controlando para diagnóstico, corroborando as hipóteses teóricas. Os Principios de Essência, que representam a avaliação qualitativa do risco da doença, extraindo o significado principal do problema, foi preditor de maior frequência de BES $[F(130,4)=26,56, p<0,001$; $\left.\mathrm{R}^{2}=0,44\right]$ e melhor funcionalidade no dia a dia FD $\left[F(130,4)=31,26, p<0,001 ; R^{2}=0,48\right]$. Já a avaliação quantitativa do Risco Específico, que identifica risco literal da doença, foi preditor de maior frequência de PS $\left[F(130,4)=23,39, p<0,001 ; R^{2}=0,41\right]$ e $R\left[F(130,4)=7,90, p<0,001 ; R^{2}=0,18\right]$.

Tabela 2 - Regressão Linear Múltipla para Predizer Sofrimento Psicológico de acordo com o Clinical Outcome Routine Evaluation - Outcome Measure

\begin{tabular}{|c|c|c|c|c|}
\hline & $B$ & SE & b & $t$ \\
\hline \multicolumn{5}{|l|}{ CORE-OM Escore Total } \\
\hline Constante & 1,91 & 0,52 & & $3,64^{* *}$ \\
\hline Diagnóstico COVID-19 & 1,09 & 0,10 & 0,70 & $11,26^{* *}$ \\
\hline Sofrimento Psicológico Significativo & 0,02 & 0,04 & 0,02 & 0,39 \\
\hline Princípios de Essência & $-0,23$ & 0,11 & $-0,14$ & $-2,18^{*}$ \\
\hline Risco Específico & 0,09 & 0,04 & 0,14 & $2,26^{*}$ \\
\hline \multicolumn{5}{|l|}{ Bem-Estar Subjetivo } \\
\hline Constante & 2,85 & 0,75 & & $3.79^{* *}$ \\
\hline Diagnóstico COVID-19 & 1,42 & 0,14 & 0,68 & $10,22^{* *}$ \\
\hline Sofrimento Psicológico Significativo & 0,03 & 0,06 & 0,04 & 0,58 \\
\hline Princípios de Essência & $-0,31$ & 0,15 & $-0,13$ & $-2,01^{*}$ \\
\hline Risco Especifico & 0,04 & 0,06 & 0,05 & 0,74 \\
\hline \multicolumn{5}{|l|}{ Problemas e Sintomas } \\
\hline Constante & 1,74 & 0,74 & & $2,33^{*}$ \\
\hline Diagnóstico COVID-19 & 1,28 & 0,14 & 0,63 & $9,32^{* *}$ \\
\hline Sofrimento Psicológico Significativo & 0,04 & 0,06 & 0,05 & 0,75 \\
\hline Princípios de Essência & $-0,15$ & 0,15 & $-0,07$ & $-0,97$ \\
\hline Risco Específico & 0,13 & 0,06 & 0,15 & $2,24^{*}$ \\
\hline
\end{tabular}




\begin{tabular}{|c|c|c|c|c|}
\hline & $B$ & SE & b & $t$ \\
\hline \multicolumn{5}{|l|}{ Funcionalidade Diária } \\
\hline Constante & 3,04 & 0,61 & & $4.97^{* *}$ \\
\hline Diagnóstico COVID-19 & 1,21 & 0,11 & 0,68 & $10,66^{* *}$ \\
\hline Sofrimento Psicológico Significativo & $-0,03$ & 0,05 & $-0,05$ & $-0,71$ \\
\hline Princípios de Essência & $-0,45$ & 0,13 & $-0,23$ & $-3,58^{* \star}$ \\
\hline Risco Especifico & 0,09 & 0,05 & 0,12 & $1,89^{+}$ \\
\hline \multicolumn{5}{|l|}{ Risco } \\
\hline Constante & 0,00 & 0,51 & & 0,00 \\
\hline Diagnóstico COVID-19 & 0,45 & 0,09 & 0,39 & $4,81^{* \star}$ \\
\hline Sofrimento Psicológico Significativo & 0,02 & 0,04 & 0,04 & 0,52 \\
\hline Princípios de Essência & $-0,03$ & 0,10 & $-0,02$ & $-0,28$ \\
\hline Risco Específico & 0,11 & 0,04 & 0,22 & $2,68^{*}$ \\
\hline
\end{tabular}

${ }^{+} p<, 10,{ }^{*} p<, 05,{ }^{* *} p<, 01$

Percepção dos participantes com maior sofrimento psicológico

Os participantes ainda foram classificados quanto a presença e ausência de sofrimento psicológico significativo. O critério adotado para a inclusão no grupo com sofrimento psicológico significativo envolvia o endosso há pelo menos uma questão da escala de Risco do CORE-OM e, concomitantemente, a presença de escores superiores ao percentil 90\% na escala Não Risco do CORE-OM, em comparação a estudo com maior tamanho amostral. Esses critérios avaliam, respectivamente, a presença de risco e de sofrimento mental e, quando usados em combinação, aumenta a probabilidade de identificação de pessoas com potencial psicopatologia. A partir disso, foram considerados 19 participantes no grupo de maior sofrimento psicológico. Esse grupo foi composto por 15 mulheres $(78,9 \%) \mathrm{com}$ média de idade de 40,89 anos ( $D P=12,01$ ).

Os resultados da análise de regressão logística binária e hierárquica, para criar um modelo preditivo de sofrimento psicológico significativo e endosso a risco, apresentou índices adequados de ajuste $\left[x^{2}(17)=31,041 ; p=0,02 ; R^{2}\right.$ Nagelkerke = 0,399]. Das variáveis demográficas e de saúde incluídas no primeiro bloco de análise, apenas o histórico de atendimento psiquiátrico ou psicológico restou significativa ( $p=0,014 ;$ OR=6,93 [IC95\%=1,474-32,671]), enquanto sexo, idade e estado civil não contribuíram para o modelo. Já no segundo passo de análise, foram inseridas as percepções quantitativas e qualitativas de riscos. Nessa etapa, foram observados efeitos significativos nas seguintes questões de representação qualitativa (Principios de Essência): "De vez em quando é bom divertir-se sem pensar nas consequências" ( $p=0,039$; $\mathrm{OR}=0,326$ [IC95\%=0,113-0,943]), "Melhor se proteger do que arriscar o contágio da COVID-19" ( $p=0,013$; $\mathrm{OR}=0,310$ [IC95\%=0,001-0,376]). Além de um item da escala quantitativa Risco Especifico: "É provável que eu tenha sintomas da COVID-19 até o final do ano" ( $p=0,048 ;$ OR=0,334 [IC95\%=0,113-0,991].

\section{Discussão}

Por estarem na linha de frente no enfrentamento da COVID 19, os profissionais de saúde são altamente vulneráveis ao sofrimento (Serrano-Ripoll et al., 2020; Busch et al., 2021). No entanto, a experiência e os comportamentos destes profissionais diante do estressor são variadas. Relatos comuns difundidos nas mídias indicam que, em alguns casos, há cuidados exacerbados de saúde, como sair de sua casa para residir em hotel, a fim de proteger a familia; em outros, há comportamentos de negligência, como uso inadequado de EPIs, que conduziram ao aumento da contaminação no meio da classe médica e 
da saúde. Segundo hipóteses da Teoria do Traço Difuso (Reyna, 2012; Reyna \& Brainerd, 2011), essas reações contraditórias estão relacionadas à forma como as informações são processadas: ancoradas em um extremo pela representação qualitativa que resume a essência do significado da situação de risco, como "Melhor prevenir do que remediar"; e, no outro extremo, pela representação quantitativa que envolve negociação entre riscos e benefícios, levando a um senso de vulnerabilidade, como "Eu vou contrair a doença no próximo mês", assumindo a noção que isso acontecerá "mesmo que eu tome precauções", e, portanto, aumentando a exposição ao risco.

Segundo a Teoria do Traço Difuso, os comportamentos de proteção e negligência frente à doença dependem da interpretação das "pistas" que desencadeiam o processamento de essência ou literal, respectivamente (Brust-Renck et al., 2016; Reyna, 2012). Uma representação mental da experiência de forma qualitativa tende a ser protetiva de risco uma vez que o significado de tais riscos (e.g., COVID-19) é que eles são "ruins" e, portanto, "devem ser evitados". As pessoas que preferem pensar de forma quantitativa, utilizando uma base de representação mental que contrabalança riscos (da doença) e beneficios (das medidas de cuidado à saúde), tendem a buscar o risco (e.g., uso inadequado de EPIs) porque estima de forma incorreta a probabilidade de contágio (Reyna, 2020a, 2020b). O presente trabalho corrobora essas hipóteses, de forma que o endosso às variáveis qualitativas, buscando compreender a essência da doença, foi associado a menor sofrimento mental (maior bem-estar subjetivo e funcionalidade diária, e provavelmente ao maior cuidado de saúde, que contribuiu para aliviar o sofrimento. Já o endosso às variáveis quantitativas, indicando capacidade de extrair probabilidades exatas sobre a chance de contaminação, sem interpretação à luz de cuidados da saúde, foi associado a maior sofrimento mental (problemas e sintomas, e risco), indicando tendência a expressar comportamentos de agressão para si e para os outros. Nesse caso, o sofrimento mental pode ter interferido na capacidade de reconhecer a essência de quando uma ameaça está presente, levando a dependência das probabilidades especificas (literais) do risco (Reyna \& Landa, 2015)

Os profissionais de saúde encontram grandes desafios no combate e tratamento da doença, depararam-se com incertezas e vulnerabilidades, diante de um vírus ainda pouco conhecido, com medo de infectar a si e a sua familia, a partir das mudanças no ambiente de trabalho e a necessidade de isolamento social. As dificuldades encontradas no enfrentamento da pandemia ficaram mais explícitas com o despreparo dos sistemas governamentais e de saúde em ofertar apoio aos profissionais da linha de frente. Ademais, as jornadas de trabalho se tornaram mais exaustivas, a falta de informações sobre o vírus e a inconsistência de recomendações oficiais contribuíram diretamente para o agravamento de muitos sintomas psicopatológicos. Proteger a integridade física e a saúde mental desses profissionais são necessidades prementes e indissociáveis. Neste sentido, os dados do presente estudo contribuem para que se planejem ações preventivas em saúde mental com essa população levando em consideração, entre outros aspectos, o modo como se dá a avaliação do risco por parte desses profissionais.

O modelo preditivo para classificação de profissionais da saúde no subgrupo que refere risco psicológico (a si e aos outros) e, simultaneamente, escores altos de sofrimento mental, também corrobora estudos prévios. Assim como neste estudo, a presença de histórico de transtornos mentais foi reportada como risco para sofrimento psicológico em um estudo similar, conduzido com trabalhadores de uma universidade do Brasil. Além disso, uma revisão sistemática também indicou esse fator como risco para diversos desfechos negativos na saúde mental de profissionais da saúde, durante a pandemia COVID-19 e em situações análogas (Preti et al., 2020). Essa mesma revisão indicou que variáveis relacionadas ao risco e proteção no contexto de trabalho também aumentam ou protegem de sofrimento mental os profissionais de saúde. Nesse sentido, os escores mais altos em algumas questões relacionadas 
aos Principios de Essência, também implicaram na redução da chance de ser classificado nesse subgrupo com indicadores clínicos. Ou seja, a Teoria do Traço Difuso também apoiou a identificação de profissionais em risco de psicopatologia.

\section{Considerações finais}

Na vigência de uma pandemia com tal magnitude, a proteção à saúde mental dos profissionais que atuam na linha de frente é essencial, não apenas como cuidado direto à um grupo mais vulnerável, mas também como medida de cuidado indireto à população, que depende da prestação dos serviços essenciais por eles prestados. Estudos sobre os fatores que impactam a saúde mental são, portanto, cruciais para somar tanto aos esforços de controle da pandemia como de proteção ao sofrimento psicológico decorrentes da mesma. O estudo sugere que a falta de uma compreensão qualitativa do risco, segundo a Teoria do Traço Difuso, pode ser responsável pela exposição dos profissionais de saúde de linha de frente a situações de risco no combate à COVID-19. O modelo teórico já foi utilizando com sucesso a intervenções de prevenção e mudança de comportamento em adolescentes e adultos jovens não sintomáticos, bem como em pacientes adultos (para uma revisão, ver Blalock \& Reyna, 2016). O histórico de aplicações bem-sucedidas da teoria na redução de comportamentos de risco sugere um bom prognóstico para o desenvolvimento de futuras práticas preventivas imediatas com essa população, bem como implicações para a psicoterapia (e.g., Reyna \& Landa, 2015).

\section{Referências}

Badahdah, A., Khamis, F., Al Mahyijari, N., Al Balushi, M., Al Hatmi, H., Al Salmi, I., Albulushi, Z., \& Al Noomani, J. (2021). The mental health of health care workers in Oman during the COVID-19 pandemic. International Journal of Social Psychiatry, 67(1), 90-95. https://doi. org/10.1177/0020764020939596

Blalock, S. J., \& Reyna, V. F. (2016). Using fuzzy-trace theory to understand and improve health judgments, decisions, and behaviors: A literature review. Health Psychology, 35(8), 781-792. https://doi.org/10.1037/hea0000384
Blake, H., Gupta, A., Javed, M., Wood, B., Knowles, S., Coyne, E., \& Cooper, J. (2021). COVID-Well Study: Qualitative Evaluation of Supported Wellbeing Centres and Psychological First Aid for Healthcare Workers during the COVID-19 Pandemic. International Journal of Environmental Research and Public Health, 18(7), Article 3626. https://doi.org/10.3390/ijerph18073626

Brust-Renck, P. G., Reyna, V. F., Wilhelms, E. A., \& Lazar, A. N. (2016). A fuzzy-trace theory of judgment and decision-making in health care: Explanation, prediction, and application. In M. A. Diefenbach, S. Miller-Halegoua, \& D. J. Bowen (Eds.), Handbook of Health Decision Science (pp. 71-86). https://doi.org/10.1007/978-1-4939-3486-7_6

Busch, I. M., Moretti, F., Mazzi, M., Wu, A. W., \& Rimondini, M. (2021). What we have learned from two decades of epidemics and pandemics: A systematic review and meta-analysis of the psychological burden of frontline healthcare workers. Psychotherapy and PsychosomatiCS, 90(3), 178-190. https://doi.org/10.1159/000513733

Chung, J. P., \& Yeung, W. S. (2020). Staff mental health self-assessment during the COVID-19 outbreak. East Asian Archives of Psychiatry, 30(1), 34. https://doi. org/10.12809/eaap2014

Conselho Federal de Enfermagem (9 de março de 2021). Brasil perde ao menos um profissional de saúde a cada 19 horas para a Covid. http:// www.cofen.gov.br/brasil-perde-ao-menos-umprofissional-de-saude-a-cada-19-horas-para-acovid_85778.html

Conselho Federal de Psicologia (26 de junho de 2012). Resolução de fiscalização e orientação [CFP, no 10/2012]. Dispõe sobre a realização de pesquisa em Psicologia com seres humanos. https://atosoficiais.com.br/cfp/resolucaode-fiscalizacao-e-orientacao-n-10-2012-revogaa-resolucao-cfp-n-0162000-publicadano-dou-n-246-secao-1-pagina-91-no-dia-22-dedezembro-de-2000-que-dispoe-sobre-a-realizacao-de-pesquisa-em-psicologia-com-seres-humanos

Conselho Nacional de Saúde. (13 junho 2013). Resolução do Plenário do Conselho Nacional de Saúde [n॰ 466, de 12 de dezembro de 2012]. Dispõe sobre diretrizes e normas regulamentadoras de pesquisas envolvendo seres humanos. Diário Oficial da República Federativa do Brasil. http://conselho.saude.gov.br/ resolucoes/2012/Reso466.pdf

Conselho Nacional de Saúde (24 de maio de 2016) Resolução do Plenário do Conselho Nacional de Medicina [n 510 de 07 de abril de 2016]. Dispõe sobre as normas aplicáveis a pesquisas em Ciências Humanas e Sociais. Diário Oficial da República Federativa do Brasil. http://bit.ly/2fmnKeD

Dong, L., \& Bouey, J. (2020). Public Mental Health Crisis during COVID-19 Pandemic, China. Emerging Infectious Diseases, 26(7), 1616-1618. https://doi.org/10.3201/ eid2607.200407

Evans, C., Connell, J., Barkham, M., Margison, F., McGrath, G., Mellor-Clark, J., \& Audin, K. (2002). Towards a standardised brief outcome measure: Psychometric properties and utility of the CORE-OM. British Journal of Psychiatry, 180(1), 51-60. https://doi.org/10.1192/bjp.180.1.51 
Evans, C., Mellor-Clark, J., Margison, F., Barkham, M., Audin, K., Connell, J., \& McGrath, G. (2000). CORE: Clinical Outcomes in Routine Evaluation. Journal of Mental Health, 9(3), 247-255.

Giorgi, G., Lecca, L. I., Alessio, F., Finstad, G. L., Bondanini, G., Lulli, L. G., Arcangeli, G., \& Mucci, N. (2020). COVID-19-Related Mental Health Effects in the Workplace: A Narrative Review. International Journal of Environmental Research and Public Health, 17(21), Article 7857. https:// doi.org/10.3390/ijerph17217857

Gold, J. A. (2020). Covid-19: adverse mental health outcomes for healthcare workers. BMJ, 369. Article m1815. https://doi.org/10.1136/bmi.m1815

Lai, J., Ma, S., Wang, Y., Cai, Z., Hu, J., Wei, N., Wu, J., Du, H., Chen, T., Li, R., Tan, H., Kang, L., Yao, L., Huang, M., Wang, H., Wang, G., Liu, Z., Hu, S. (2020). Factors associated with mental health outcomes among health care workers exposed to coronavirus disease 2019. JAMA Netw Open, 3(3), Article e203976. https://doi. org/10.1001/jamanetworkopen.2020.3976

Lara, P. G., Silva, G. R., Servino, L. L., Frimm, V. C., \& Kristensen, C. H. (2019). Primeiros socorros psicológicos: Intervenção em crise para eventos de violência urbana. Revista Educar Mais, 3(3), 9-16. https://doi.org/10.15536/ reducarmais.3.2019.9-16.1607

Mills, B., Reyna, V. F., \& Estrada, S. (2008). Explaining contradictory relations between risk perception and risk taking. Psychological Science, 19(5), 429-433. https:// doi.org/10.1111/j.1467-9280.2008.02104.X

Nacoti, M., Ciocca, A., Giupponi, A., Brambillasca, P., Lussana, F., Pisano, M., Goisis, G., Bonacina, D., Fazzi, F., Naspro, R., Longhi, L., Cereda, M., \& Montaguti, C. (2020). At the epicenter of the Covid-19 pandemic and humanitarian crises in Italy: Changing perspectives on preparation and mitigation. NEJM Catalyst Innovations in Care Delivery, 1(2), 1-5. https://doi.org/10.1056/CAT.20.0080

Ornell, F., Halpern, S. C., Kessler, F. H. P., \& Narvaez, J. C. M. (2020). The impact of the COVID-19 pandemic on the mental health of healthcare professionals. Cadernos de Saúde Pública, 36(4), Article e00063520. https://doi. org/10.1590/0102-311x00063520

Preti, E., Di Mattei, V., Perego, G., Ferrari, F., Mazzetti, M., Taranto, P., Di Pierro, R., Madeddu, F., \& Calati, R. (2020). The psychological impact of epidemic and pandemic outbreaks on healthcare workers: rapid review of the evidence. Current Psychiatry Reports, 22(8), 1-22. https:// doi.org/10.1007/s11920-020-01166-z

Reyna, V. F. (2012). A new intuitionism: Meaning, memory, and development in Fuzzy-Trace Theory. Judgment and Decision Making, 7(3), 332-359. http://journal.sjdm. org/11/111031/jdm111031.pdf

Reyna, V. F. (2020a). A scientific theory of gist communication and misinformation resistance, with implications for health, education, and policy. Proceedings of the $\mathrm{Na}$ tional Academy of Sciences, 118(15), Article e1912441117. https://doi.org/10.1073/pnas.1912441117

Reyna, V. F. (2020b). Of viruses, vaccines, and variability: Qualitative meaning matters. Trends in Cognitive Sciences, 24(9), 672-675. https://doi.org/10.1016/j. tics.2020.05.015
Reyna, V. F., \& Brainerd, C. J. (2011). Dual processes in decision making and developmental neuroscience: A fuzzy-trace model. Developmental Review, 31, 180-206. https://doi.org/10.1016/j.dr.2011.07.004

Reyna, V. F., \& Landa, Y. (2015). Multiple traces or fuzzy traces? Converging evidence for applications of modern cognitive theory to psychotherapy. Behavioral and Brain Sciences, 38(22), 39. https://doi.org/10.1017/ $\underline{\mathrm{S} 0140525 \times 14000399}$

Ruiz, M. A., \& Gibson, C. A. M. (2020). Emotional impact of the COVID-19 pandemic on US health care workers: A gathering storm. Psychological Trauma: Theory, Research, Practice, and Policy, 12(S1), S153-S155. https:// doi.org/10.1037/tra0000851

Saidel, M., Lima, M., Campos, C., Loyola, C., Espiridião, E., \& Rodrigues, J. (2020). Intervenções em saúde mental para profissionais de saúde frente a pandemia de Coronavirus. Revista Enfermagem UERJ, 28, Article e49923. https://doi.org/10.12957/reuerj.2020.49923

Sani, G., Janiri, D., Di Nicola, M., Janiri, L., Ferretti, S., \& Chieffo, D. (2020). Mental health during and after the COVID-19 emergency in Italy. Psychiatry and Clinical Neurosciences, 74(6), 372. https://doi.org/10.1111/pcn.13004

Santana, M. R. M.; Da Silva, M. M.; De Moraes, D. S.; Fukuda, C. C.; Freitas, L. H.; Ramos, M. E. C.; Fleury, H. J.; Evans, C. (2015). Brazilian Portuguese version of the CORE-OM: Cross-cultural adaptation of an instrument to assess the efficacy and effectiveness of psychotherapy. Trends Psychiatry Psychotherapy. 37, 227-231.

Serrano-Ripoll, M. J., Meneses-Echavez, J. F., Ricci-Cabello, I., Fraile-Navarro, D., Fiol-deRoque, M. A., Pastor-Moreno, G., Castro, A., Ruiz-Pérez, I., Campos, R. Z., \& Gonçalves-Bradley, D. C. (2020). Impact of viral epidemic outbreaks on mental health of healthcare workers: A rapid systematic review and meta-analysis. Journal of Affective Disorders, 277, 347-357. https://doi. org/10.1016/j.jad.2020.08.034

Secretaria da Saúde do Estado do Rio Grande do Sul (3 de março de 2021). Painel Coronavirus RS. https://saude.rs.gov.br/secretariada-saude-arita-bergmann-fala-sobre-o-esgotamento -dos-leitos-de-uti-no-rs?midia=secretariada-saude-arita-bergmann-fala-sobre-oesgotamento-dos-leitos-de-uti-no-rs

Talevi, D., Socci, V., Carai, M., Carnaghi, G., Faleri, S., Trebbi, E., di Bernardo, A., Capelli, F., \& Pacitti, F. (2020). Mental health outcomes of the COViD-19 pandemic. Rivista di psichiatria, 55(3), 137-144. https://doi. org/10.1708/3382.33569

\section{Priscila Goergen Brust-Renck}

Doutora e mestre em Developmental Psychology pela Cornell University, em Ithaca, NY, Estados Unidos da América; mestre em Psicologia, com ênfase em Cognição Humana, pela Pontifícia Universidade Católica de Porto Alegre (PUCRS), em Porto Alegre, RS, Brasil; professora do Programa de Pós-Graduação em Psicologia da Universidade do Vale do Rio dos Sinos (UNISINOS), em São Leopoldo, RS, Brasil. 


\section{Jocieli Ferrari}

Mestranda em Psicologia pelo Programa de Pós-Graduação em Psicologia da Universidade do Vale do Rio dos Sinos (UNISINOS), em São Leopoldo, RS, Brasil; coordenadora do Serviço Especializado de Psicologia do Saúde Univates da Universidade do Vale do Taquari (UNIVATES), em Lajeado, RS, Brasil.

\section{Murilo Ricardo Zibetti}

Doutor e mestre em Psicologia pela Universidade Federal do Rio Grande do Sul (UFRGS), em Porto Alegre, RS, Brasil; professor do Programa de Pós-Graduação em Psicologia da Universidade do Vale do Rio dos Sinos (UNISINOS), em São Leopoldo, RS, Brasil.

\section{Fernanda Barcellos Serralta}

Doutora em Psiquiatria e Ciências do Comportamento pela Universidade Federal do Rio Grande do Sul (UFRGS), em Porto Alegre, RS, Brasil; mestre em Psicologia pela Pontifícia Universidade Católica de Porto Alegre (PUCRS), em Porto Alegre, RS, Brasil; professora do Programa de Pós-Graduação em Psicologia da Universidade do Vale do Rio dos Sinos (UNISINOS), em São Leopoldo, RS, Brasil.

\section{Endereço para correspondência}

Priscila Goergen Brust-Renck

Universidade do Vale do Rio dos Sinos

Av. Unisinos, 950, Sala E02 116

Cristo Rei, 93022-750

São Leopoldo, RS, Brasil

Os textos deste artigo foram revisados pela Poá Comunicação e submetidos para validação do(s) autor(es) antes da publicação. 